Dijk, G.M. van, Veenhof, C., Lankhorst, G.J., Dekker, J. Limitations in activities in patients with osteoarthritis of the hip or knee: the relationship with body functions, comorbidity and cognitive functioning Disability and Rehabilitation: 2009, 31(20), 1685-1691

\begin{tabular}{|l|l|}
\hline $\begin{array}{l}\text { Postprint } \\
\text { Version } \\
\text { Journal website }\end{array}$ & 1.0 \\
\hline Pubmed link & $\underline{\underline{\text { http://www.informaworld.com/smpp/content } \sim \mathrm{db}=\text { all?content }=10.1080 / 0963828}}$ \\
\hline DOI & $\underline{\mathrm{http} / / / \mathrm{www} . n \text { cbi.nlm.nih.gov/pubmed/19479564 }}$ \\
\hline
\end{tabular}

This is a NIVEL certified Post Print, more info at http://www.nivel.eu

\title{
Limitations in activities in patients with osteoarthritis of the hip or knee: the relationship with body functions, comorbidity and cognitive functioning
}

GABRIELla M. VAN DIJK ${ }^{1}$, PT, MSC, CINDY VEENHOF ${ }^{1}$, PT, PHD, GUUS J. LANKHORST ${ }^{2}$, PHD, JOOST DEKKER ${ }^{2}$, PHD.

1 NIVEL (Netherlands Institute for Health Services Research), Utrecht, The Netherlands;

2 Department of Rehabilitation Medicine, EMGO Institute, VU University Medical Centre Amsterdam, The Netherlands.

\section{Abstract}

Purpose: To determine the relationship between body functions, comorbidity and cognitive functioning on the one side and limitations in activities on the other, in elderly patients with OA of the hip or knee.

Method: A cross-sectional cohort study was conducted in which 288 patients with hip or knee OA were included. Patients were recruited from rehabilitation centres and hospitals (departments of Orthopedics, Rheumatology or Rehabilitation). Apart from demographic and clinical data, information about limitations in activities, body functions (pain, muscle strength, range of joint motion), comorbidity and cognitive functioning was collected by questionnaires and tests. Statistical analyses included univariate and stepwise multivariate regression analysis.

Results: Self-reported limitations in activities (WOMAC) were significantly associated with pain, muscle strength knee extension, ROM hip flexion and morbidity count. Performance-based limitations in activities (timed walking test) were significantly associated with ROM (knee flexion, hip flexion, and knee extension), muscle strength hip abduction, pain, cognitive functioning and age.

Conclusions: Self-reported limitations in activities in hip or knee OA are largely dependent on pain and to a lesser extent on range of joint motion, muscle strength and comorbidity. Performance-based limitations in activities are largely dependent on range of joint motion and muscle strength, and to a lesser extent on pain, cognitive functioning and other factors. These findings point to the role of body functions in limitations in activities in OA of the hip or knee. Although less important, comorbidity and cognitive functioning play a role as well. 
Dijk, G.M. van, Veenhof, C., Lankhorst, G.J., Dekker, J. Limitations in activities in patients with osteoarthritis of the hip or knee: the relationship with body functions, comorbidity and cognitive functioning Disability and Rehabilitation: 2009, 31(20), 1685-1691

\section{INTRODUCTION}

Osteoarthritis (OA), a common disease in elderly people, is characterized by progressive radiological changes, pain and limitations in activities. Furthermore, physical impairments such as reduced muscle strength and restricted range of motion (ROM) are often observed (1-3). Radiological impairments, pain and muscle strength have been frequently studied as possible determinants of limitations in activities. Also ROM has received some attention in studies on limitations in activities. Results suggested that there is no or only a weak association between radiological impairments and disability (4). Pain and reduced muscle strength are associated with increased limitations in activities. Restricted joint mobility appears to be an important determinant as well (1-4).

It is assumed that in elderly patients with OA, limitations in activities are also influenced by comorbidity and cognitive functioning. $\mathrm{OA}$ is one of diseases with the highest rate of comorbidity (5-7) and there is evidence that comorbidity is related to disability $(6 ; 8-10)$. Cognitive impairments are frequently seen in the elderly, but their prevalence and association with limitations in activities has not yet been studied in patients with OA.

Although these factors have been studied separately, it is not known whether comorbidity and cognitive functioning contribute to limitations in activities, in addition to the well known effects of body functions (muscle strength, range of joint motion) and pain. The objective of the study was to assess the relationship between body functions, comorbidity and cognitive functioning on the one side and limitations in activities on the other side, in elderly patients with hip or knee OA.

\section{METHODS}

\section{Design}

A cross-sectional study was conducted in 288 patients with knee or hip OA. The study was approved by the Medical Ethical Committee of the VU University medical centre, Amsterdam, the Netherlands.

\section{Study population}

Patients were recruited from three rehabilitation centres and two hospitals (departments of Orthopaedics, Rheumatology or Rehabilitation). The present study is part of a larger research program on rehabilitation of elderly patients with OA of the hip or knee. For this reason, we focussed on rehabilitation centres and hospitals in recruiting patients. Inclusion criteria were: (a) diagnosis of hip or knee OA by medical specialist according to radiological criteria or clinical criteria of the American College of Rheumatology (11;12); (b) 50 years of age or older; (c) referral to hospital or rehabilitation centre due to osteoarthritis of the hip or knee, less than one year before inclusion; (d) at least moderate functional problems (Lequesne algofunctional index score $\geq 5$ ) (13) and (e) informed consent. Exclusion criteria were: (a) 85 years of age or older; (b) insufficient understanding of the Dutch language and (c) expected death within one year after inclusion, due to fatal illness.

Initially, 775 patients with osteoarthritis of the hip or knee that visited the department in the year prior to inclusion were contacted by mail and were asked to participate in the study. Of those patients that volunteered $(\mathrm{n}=364), 288$ were included. 76 patients were excluded. Reasons of exclusion were age $(n=2)$, language $(n=4)$, less than moderate functional problems $(n=48)$ and referral longer than one year before inclusion $(n=22)$. No differences were found between the group of patients that were initially contacted $(\mathrm{N}=775)$ and the patients that were included in the study $(\mathrm{N}=288)$ with regard to age and gender. Compared to our study population, patients that were initially contacted suffered less frequently from both hip and knee OA $(6.2 \%$ versus $26.5 \%)$ and more frequently from knee OA $(59.5 \%$ versus $48.4 \%)$ and hip OA (34.3\% versus $25.1 \%)$. 
Dijk, G.M. van, Veenhof, C., Lankhorst, G.J., Dekker, J. Limitations in activities in patients with osteoarthritis of the hip or knee: the relationship with body functions, comorbidity and cognitive functioning Disability and Rehabilitation: 2009, 31(20), 1685-1691

\section{Measurements}

Measurements were carried out by tests, questionnaires and interviews. Assessments were performed on test locations by the researcher or the research assistant.

\section{Demographic and clinical data.}

Demographic and clinical data were collected including age, gender, height, weight, location of OA, duration of complaints, other joint complaints, radiological impairments, level of education and marital state. Body Mass Index (BMI) was calculated; obesity was defined as BMI $>30$. If available, $X$-rays of the hip and knee that were recorded in the year prior to inclusion were scored on joint space width and osteophytes, following a standardized procedure (14). A $0-3$ scale was used for rating the radiographs: $0=$ normal; $1=$ mild or $1-33 \%$ abnormal; $2=$ moderate or $34-66 \%$ abnormal; $3=$ severe or $67-100 \%$ abnormal. Frome these scores Kellgren \& Lawrence grades were calculated.

\section{Limitations in activities.}

Self-reported limitations in activities were measured using the physical functioning subscale of the Western Ontario and McMaster Universities Osteoarthritis Index (WOMAC) $(15 ; 16)$. Standardized scores of the WOMAC, ranging from 0 to 100 were used. A higher score on WOMAC reflects fewer limitations. Performance-based limitations in activities were measured using a 10 meter timed walking test (17). A higher score on the timed walking test reflects more limitations.

\section{Body functions.}

Assisted active range of Motion (ROM) was measured in both legs using goniometry, following a standardized protocol (18). For the hip, internal rotation, external rotation and flexion were measured. For the knee, flexion and extension were measured. Isometric muscle strength of knee extension and hip abduction was measured in both legs with a hand held dynamometer, the MicroFet. A standardized protocol was used (19). The measurements of both ROM and muscle strength were repeated twice. The average score was used in the analyses. Patients rated their pain at the time of the assessment on a Visual Analogue Scale (VAS). A higher score on the VAS reflects more pain.

\section{Comorbidity.}

Information about comorbidity was gathered in an interview with the patients using the Cumulative Illness Rating Scale (CIRS) (20;21). The CIRS consists of 13 body systems. Scoring is weighted by the severity of the comorbid condition. Severity scores range from 0 (none) to 4 (extremely severe). For detailed information about the CIRS, the reader is referred to an earlier study (10). Because all patients suffered from OA and this disease category can be regarded as the index disease, diseases in CIRS10 (muscle, bone and skin diseases) were excluded from the analyses. The index of comorbidity that was derived from the CIRS is morbidity count, the number of diseases on which the patients scored a severity of 2 or higher was calculated.

\section{Cognitive functioning.}

Various aspects of cognitive function were measured. Firstly, the 20 item cognitive screening test (CST20) was applied. This instrument was developed as a screening instrument for cognitive decline in elderly (22). Scores range from 0 to 20. An indication of cognitive decline is calculated. For patients older than 81 years, cognitive decline is defined by a score on the CST20 of 10 or less, for patients of 81 years or younger, cognitive decline is defined by a score on the CST20 of 12 or less. Secondly, a memory test, extracted from the Wechsler Adult Intelligence Scale (WAIS), was applied (23;24). Patients were asked to recall series of numbers, both backward and forward. The score was calculated by the number of correctly repeated items. Backward and forward scores range from 0 to 21 . Finally, to assess visual selective attention, the abridged Stroop Color Word test was applied $(25 ; 26)$. The variables derived from the Stroop test are the number of uncorrected mistakes 
Dijk, G.M. van, Veenhof, C., Lankhorst, G.J., Dekker, J. Limitations in activities in patients with osteoarthritis of the hip or knee: the relationship with body functions, comorbidity and cognitive functioning Disability and Rehabilitation: 2009, 31(20), 1685-1691

in part III of the test and the interference score (the time needed for part III minus the time needed for part II).

\section{Statistical analyses}

Following the method used by Steultjens et al. (1;2), indices for ROM (hip flexion, hip external rotation, hip internal rotation, knee extension and knee flexion) and muscle strength (hip abduction and knee extension) were calculated using the sum score for left and right for each movement. Prior to this calculation, scores for muscle strength on the Microfet were divided by body weight.

For radiological impairments, a combined score for the hip and the knee was calculated. For the multivariate regression analyses radiological impairments of the knee and radiological impairments of the hip were regarded positive if patients showed severe joint space narrowing or severe osteophytes in either the hip or the knee (rating $=3$ ). Any severe abnormality resulted in a positive score on this variable.

For body functions, comorbidity and cognitive functioning, univariate regression analyses were performed with WOMAC physical functioning score and the timed walking test as dependent variables. Furthermore, stepwise multiple regression analyses were performed, again using WOMAC physical functioning score and the timed walking test as dependent variables. Variables were added to the model if $p<0.05$. The following blocks of variables were entered stepwise: (1) ROM and muscle strength; (2) pain; (3) comorbidity; (4) cognitive functioning; (5) BMI and age. The 11.5 version of SPSS was used.

\section{RESULTS}

\section{Study population}

Baseline characteristics of the study population are presented in table 1 . The majority of the patients $(80 \%)$ originated from departments of Orthopaedics. The other $20 \%$ came from departments of Rheumatology and departments of Rehabilitation. On average, patients had had hip and knee complaints for 10.1 years. Only $13 \%$ had no other joint complaints. Frequently occurring other joint complaints were hand and back problems.

\section{[TABLE 1]}

\section{Univariate analyses}

Results of the univariate regression analyses are presented in table 2 . These results show that associations $(\mathrm{p}<0.05)$ were found for WOMAC physical functioning score and pain, ROM (except knee extension), muscle strength, morbidity count, numbers forward score, Stroop interference score and BMI. Furthermore, associations $(\mathrm{p}<0.05)$ were found between the timed walking test and pain, ROM, muscle strength, morbidity count, numbers forward score, Stroop interference score and age. Correlation between the WOMAC and the timed walking test was $0.423(\mathrm{p}<0.01)$.

\section{[TABLE 2]}

\section{Self-reported limitations in activities}

Table 3 shows the results of the multiple regression analyses for self-reported limitations in activities measured by the WOMAC. Factors that were found to be associated with selfreported limitations in activities were ROM hip flexion $(\beta=0.133)$, ROM hip internal rotation $(\beta=0.041)$, muscle strength knee extension $(\beta=0.128)$, pain $(\beta=-0.576)$ and morbidity count $(\beta=-0.138)$. Lower ROM, lower muscle strength, more pain and higher morbidity count (having more comorbidities) were associated with more self-reported limitations in activities on the WOMAC. In total about $50 \%$ of the variance was explained. 
Dijk, G.M. van, Veenhof, C., Lankhorst, G.J., Dekker, J. Limitations in activities in patients with osteoarthritis of the hip or knee: the relationship with body functions, comorbidity and cognitive functioning Disability and Rehabilitation: 2009, 31(20), 1685-1691

\section{[TABLE 3]}

\section{Performance-based limitations in activities}

In table 3 multiple regression analyses are shown for performance-based limitations in activities measured by the timed walking test. In this model $36 \%$ of the variance was explained. Factors that were found to be associated with performance-based limitations in activities were ROM hip flexion ( $\beta=-0.174)$, ROM knee flexion $(\beta=-0.188)$, ROM knee extension $(\beta=-0.124)$, muscle strength hip abduction $(\beta=-0.170)$, pain measured by the VAS $(\beta=0.161)$, cognitive functioning measured by the numbers forward test $(\beta=-0.106)$ and age ( $\beta=0.206)$. ). Lower ROM, lower muscle strength, more pain, worse cognitive functioning and older age were associated with less performance-based limitations in activities.

\section{DISCUSSION}

The objective of the study was to describe the relationship between body functions, comorbidity and cognitive functioning on the one side and limitations in activities on the other side in elderly patients with knee or hip OA. Self-reported limitations in activities measured by the WOMAC were found to be largely dependent on pain, since pain explained the largest part of the variance. But ROM, muscle strength and morbidity count played an important role as well. Cognitive functioning and BMI did not influence self-reported limitations in activities. Contrary to self-reported limitations in activities, performance-based limitations in activities measured by the timed walking test were primarily explained by body functions such as ROM and muscle strength. Pain, cognitive functioning and age played a role as well. Comorbidity was not related to performance-based limitations in activities.

\section{[BOX 1 AND 2]}

The differential associations for self-reported and performance-based limitations in activities, were also reported in a study on physical functioning in knee osteoarthritis: that study found that self-reported physical functioning was more influenced by pain than performance-based physical functioning (27). We found that the correlation between performance-based limitations in activities and self-reported limitations in activities is low. This strengthens the believe that self-reported limitations in activities and performance-based limitations in activities are different concepts, which are influenced by different determinants and provide additional information about limitations in activities $(28 ; 29)$

\section{Body functions.}

Limitations in activities are largely dependent on body functions, especially pain, muscle strength and ROM. Results show that pain has a clear relationship with limitations. This finding confirms earlier research $(3 ; 30)$. Similarly, the association between muscle strength and limitations in activities as found in previous studies $(1 ; 2 ; 4)$ has been confirmed. Although so far, ROM received only little attention $(1 ; 3)$, the present study showed that ROM (in particular ROM hip flexion) plays an important role in self-reported and performance-based limitations in activities.

\section{Comorbidity.}

Significant associations between self-reported limitations in activities and comorbidity were found in this study. Comorbidity appears to be a relevant factor in limitations in activities, in addition to pain, ROM and muscle strength. Comorbidity, however, was not found to be a determinant of performance-based limitations in activities, although a significant univariate association was established. Obviously, ROM and muscle strength have a larger influence on performance-based limitations in activities. 
Dijk, G.M. van, Veenhof, C., Lankhorst, G.J., Dekker, J. Limitations in activities in patients with osteoarthritis of the hip or knee: the relationship with body functions, comorbidity and cognitive functioning Disability and Rehabilitation: 2009, 31(20), 1685-1691

\section{Cognitive functioning.}

For cognitive functioning only a weak association was found with performance-based limitations in activities. Partly, this might be explained by the high level of cognitive functioning of the studies population. Another explanation is that factors with little influence were excluded from the model, because of the strong influence of pain. Results from secondary analyses (not presented) show that excluding pain from the model reveals a small influence of cognitive functioning $(\beta=0.119)$ on self-reported limitations measured by the WOMAC.

Some methodological issues need to be considered. First, patients included in this study were recruited from hospitals and rehabilitation centers. Thus, the results of the present study cannot be generalized to the general population suffering from OA. Nevertheless, the results are applicable to the group of patients, consulting in hospitals and rehabilitation centers: this is a highly relevant group. Second, this is a cross-sectional study; Causal conclusions are not warranted. The number of longitudinal studies on functional outcome in OA is limited (31). To assess determinants of future limitations in activities, a longitudinal study will be conducted, using the baseline measurements presented in the present report.

In conclusion, self-reported limitations in activities are largely dependent on pain and to a lesser extent on range of range of joint motion, muscle strength and comorbidity. Performance-based limitations in activity are largely dependent on range of joint motion and muscle strength, and to a lesser extent on pain and cognitive functioning. These findings point to (i) the role of pain, muscle strength and range of joint motion in limitations in activities in OA of the hip or the knee; although less important, comorbidity and cognitive functioning play a role as well and to (ii) the importance of distinguishing between selfreported and performance-based limitations in activities.

\section{REFERENCES}

(1) Steultjens M, Dekker J, van Baar M, Oostendorp R, Bijlsma J. Range of joint motion and disability in patients with osteoarthritis of the knee or hip. Rheumatol 2000; 39(9):955961.

(2) Steultjens M, Dekker J, van Baar M, Oostendorp R, Bijlsma J. Muscle strength, pain and disability in patientts with osteoarthritis of the knee or hip. Clin Rehab 2001; 15(3):331341.

(3) van Baar M, Dekker J, Lemmens J, Oostendorp R, Bijlsma J. Pain and disability in patients with osteoarthritis of hip or knee: the relationship with articular, kinesiological and psychological characteristics. J Rheumatol 1998; 25:125-133.

(4) Dekker J, Boot B, Woude van der L, Bijlsma J. Pain and disability in osteoarthritis: a review of behavioral mechanisms. Journal of behavioral medicine 1992; 15(2):189-214.

(5) Gabriel SE, Crowson CS, O'Fallon WM. Comorbidity in arthritis. J Rheumatol 1999; 26(11):2475-2479.

(6) Marks R, Allegrante JP. Comorbid disease profiles of adults with end-stage hip osteoarthritis. Med Sci Monit 2002; 8(4):CR305-CR309.

(7) Schellevis FG, van der Velden J, van de Lisdonk E, van Eijk JT, van Weel C. Comorbidity of chronic diseases in general practice. J Clin Epidemiol 1993; 46(5):469-473.

(8) Caporali R, Cimmino MA, Sarzi-Puttini P, Scarpa R, Parazzini F, Zaninelli A et al. Comorbid conditions in the AMICA study patients: effects on the quality of life and drug prescriptions by general practitioners and specialists. Semin Arthritis Rheum 2005; 35(1 Suppl 1):31-37.

(9) Cimmino MA, Sarzi-Puttini P, Scarpa R, Caporali R, Parazzini F, Zaninelli A et al. Clinical presentation of osteoarthritis in general practice: determinants of pain in Italian patients in the AMICA study. Semin Arthritis Rheum 2005; 35(1 Suppl 1):17-23.

(10) van Dijk GM, Veenhof C, Schellevis FG, Hulsmans H, Ahmadi N, Bakker JPJ.

Comorbidity, limitations in activities and pain in patients with osteoarthritis of the hip or knee. BMC Musculoskelet Disord. 2008; 9: 95. 
Dijk, G.M. van, Veenhof, C., Lankhorst, G.J., Dekker, J. Limitations in activities in patients with osteoarthritis of the hip or knee: the relationship with body functions, comorbidity and cognitive functioning Disability and Rehabilitation: 2009, 31(20), 1685-1691

(11) Altman R, Asch E, Bloch D, Bole G, Borenstein D, Brandt K et al. Development of criteria for the classification and reporting of osteoarthritis. Classification of osteoarthritis of the knee. Arthritis Rheum 1986; 29(8):1039-1049.

(12) Altman R, Alarcon G, Appelrouth D, Bloch D, Borenstein D, Brandt K et al. The American College of Rheumatology criteria for the classification and reporting of osteoarthritis of the hip. Arthritis Rheum 1991; 34(5):505-514.

(13) Lequesne M, Mery C, Samson M, Gerard D. Indices of severity for osteoarthritis of the hip and knee. Scand J Rheumatol 1987; 18(Suppl 65):85-89.

(14) Altman R, Hochberg M, Murphy W, Wolfe F, Lequesne M. Atlas of individual radiopgraphic features in osteoarthritis. Osteoarthritis Cartilage 1995; 3(Suppl A):3-70.

(15) Bellamy N, Buchanan W, Goldsmith C, Campbell J, Stitt L. Validation study of WOMAC: a health status instrument for measuring clinically-important patient-relevant outcomes following total hip or knee arthroplasty in osteoarthritis. J Orth Rheumatol 1988; 1:95-108.

(16) Roorda LD, Jones CA, Waltz M, Lankhorst GJ, Bouter LM, van der Eijken JW et al. Satisfactory cross cultural equivalence of the Dutch WOMAC in patients with hip osteoarthritis waiting for arthroplasty. Ann Rheum Dis 2004; 63(1):36-42.

(17) Wade D, Wood V, Heller A, Maggs J, Hewer R. Walking after stroke. Scand J Rehab Med 1987; 19:25-30.

(18) Norkin C, White D. Measurement of joint motion: a guide to goniometry. Philadelphia: FA Davis Company, 1986.

(19) Bohannon R. Muscle strength testing with hand-held dynamometers. In: Amundsen L, editor. Muscle strength testing: instrumented and non-instrumented systems. New York: Churchill Livingstone, 1990: 69-88.

(20) Conwell Y, Forbes N, Cox C, Caine E. Validation of a measure of physical Ilness Burden at autopsy: the Cumulative Illness Rating Scale. J Am Ger Soc 1993; 41:38-41.

(21) Linn BS, Linn MW, Gurel L. Cumulative Illness Rating Scale. J Am Ger Soc 1968; 16(5):622-625.

(22) De Graaf A, Deelman B, Fens J. Cognitieve Screeningstest (CST). In: Bouma A, Mulder J, Lindenboom J, editors. Neuropsychologische diagnostiek; handboek. 1996: I I1 15.

(23) Lindenboom J, Matto D. Cijferreeksen en Knox blokken als concentratietests voor ouderen. Tijdschr Gerontol Geriatr 1994; 25:63-68.

(24) Wechsler D, Stinissen J, Willems P, Coetsier P, Hulsman W. Wechsler Adult Intelligence Scale. In: Bouma A, Mulder J, Lindenboom J, editors. Neuropsychologische diagnostiek: handboek. 1996: A 1-A 28.

(25) Klein M, Ponds R, Houx P, Jolles J. Effects of test duration on age-related difference in Stroop Interference. J Clin Exp Neuropsych 1997; 18(1):77-82.

(26) Stroop J, Hammes. Stroop Kleur-Woord test. In: Bouma A, Mulder J, Lindenboom J, editors. Neuropsychologische diagnostiek. 1996: G 1-G 12.

(27) Terwee C, van der Slikke R, van Lummel R, Benink R, Meijers W, de Vet H. Self reported physical functioning was more influenced by pain than performance-based physical functioning in knee osteoarthritis patients. J Clin Epidemiol 2006; 59(7):724-731.

(28) Maly MR, Costigan PA, Olney SJ. Determinants of self-report outcome measures in people with knee osteoarthritis. Arch Phys Med Rehabil 2006; 87(1):96-104.

(29) Stratford PW, Kennedy DM. Performance measures were necessary to obtain a complete picture of osteoarthritic patients. J Clin Epidemiol 2006; 59(2):160-167.

(30) Creamer P, Lethbridge-Cejku M, Hochberg MC. Factors associated with functional impairment in symptomatic knee OA. Rheumatol 2000; 39(5):490-496.

(31) van Dijk GM, Dekker J, Veenhof C, van den Ende CHM. Course of functional status and pain in osteoarthritis of the hip and knee: A systematic review. Arthr Care Res 2007; 55(5):779-785. 
Dijk, G.M. van, Veenhof, C., Lankhorst, G.J., Dekker, J. Limitations in activities in patients with osteoarthritis of the hip or knee: the relationship with body functions, comorbidity and cognitive functioning Disability and Rehabilitation: 2009, 31(20), 1685-1691

\section{TABLES AND BOXS}

Table I. Baseline characteristics of the study population $(N=288)$.

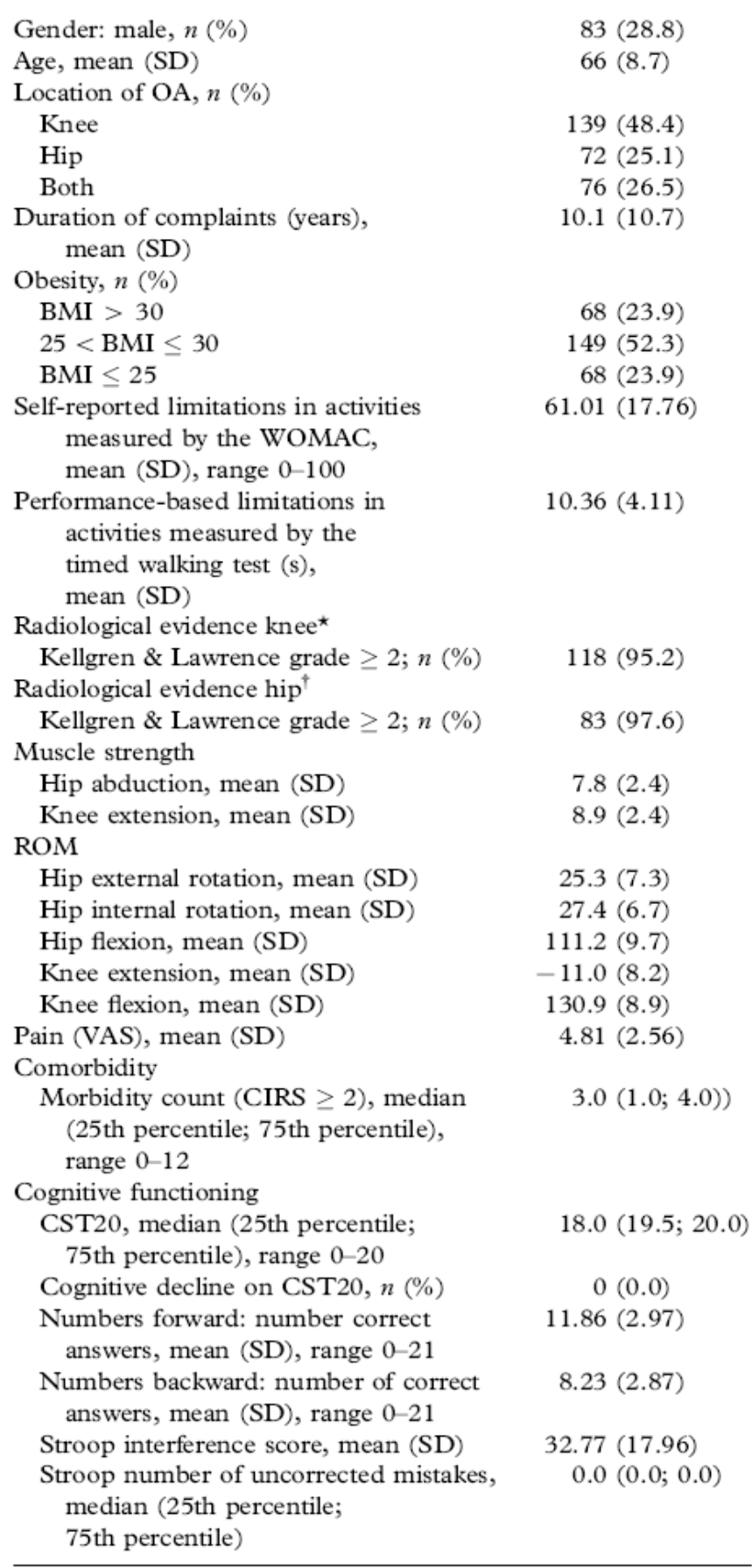

$N, n$, number; $\mathrm{SD}$, standard deviation; OA, osteoarthritis; BMI, body mass index; WOMAC, western Ontario and McMaster Universities osteoarthritis index; VAS, visual analogue scale; CIRS, cumulative illness rating scale; CST20, cognitive screening test 20 items.

${ }^{\star} N=90$ (from only a part of the included patients X-rays were available).

${ }^{\dagger} N=138$ (from only a part of the included patients X-rays were available). 
Dijk, G.M. van, Veenhof, C., Lankhorst, G.J., Dekker, J. Limitations in activities in patients with osteoarthritis of the hip or knee: the relationship with body functions, comorbidity and cognitive functioning Disability and Rehabilitation: 2009, 31(20), 1685-1691

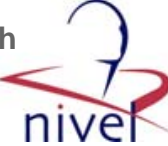

Table II. Relationship between body functions, comorbidity and cognitive functioning on the one side and limitations in activities on the other side (WOMAC and timed walking test); results of the univariate regression analyses ${ }^{\star}$.

\begin{tabular}{lcr}
\hline & $\begin{array}{c}\text { WOMAC } \\
\text { (PF) }\end{array}$ & $\begin{array}{c}\text { Timed } \\
\text { walking } \\
\text { test }\end{array}$ \\
\hline Radiological impairments & & \\
$\quad$ Radiological impairments & 0.048 & -0.027 \\
Pain & & \\
VAS pain & $-0.675^{\dagger}$ & $0.296^{\dagger}$ \\
Other body functions & & \\
ROM hip flexion & $0.376^{\dagger}$ & $-0.401^{\dagger}$ \\
ROM hip internal rotation & $0.246^{\dagger}$ & $-0.125^{\dagger}$ \\
ROM hip external rotation & $0.175^{\dagger}$ & $-0.238^{\dagger}$ \\
ROM knee flexion & $0.204^{\dagger}$ & $-0.296^{\dagger}$ \\
ROM knee extension & 0.008 & $-0.246^{\dagger}$ \\
Muscle strength hip abduction & $0.318^{\dagger}$ & $-0.356^{\dagger}$ \\
Muscle strength knee extension & $0.306^{\dagger}$ & $-0.274^{\dagger}$ \\
Comorbidity & & \\
Morbidity count (CIRS 2) & $-0.335^{\dagger}$ & $0.264^{\dagger}$ \\
Cognitive functioning & & \\
CST 20 & -0.027 & 0.012 \\
Numbers forward & $0.156^{\dagger}$ & $-0.184^{\dagger}$ \\
Numbers backward & 0.048 & -0.086 \\
Stroop interference score & $-0.184^{\dagger}$ & $0.207^{\dagger}$ \\
Stroop uncorrected mistakes score & -0.031 & $0.107^{\S}$ \\
Other factors & -0.094 & $0.204^{\dagger}$ \\
Age & $-0.229^{\dagger}$ & $0.108^{\S}$ \\
BMI & & \\
\hline
\end{tabular}

WOMAC (PF), western Ontario and McMaster Universities osteoarthritis index (physical functioning); VAS, visual analogue scale; ROM, assistive active range of motion; CIRS, cumulative illness rating scale; CST20, cognitive screening test 20 items. ${ }^{\star}$ Standardised $\beta$ 's are presented; ${ }^{\dagger} p<0.01 ;{ }^{\ddagger} p<0.05 ;{ }^{\S} 0.05<$ $p<0.1$. 
Dijk, G.M. van, Veenhof, C., Lankhorst, G.J., Dekker, J. Limitations in activities in patients with osteoarthritis of the hip or knee: the relationship with body functions, comorbidity and cognitive functioning Disability and Rehabilitation: 2009, 31(20), 1685-1691
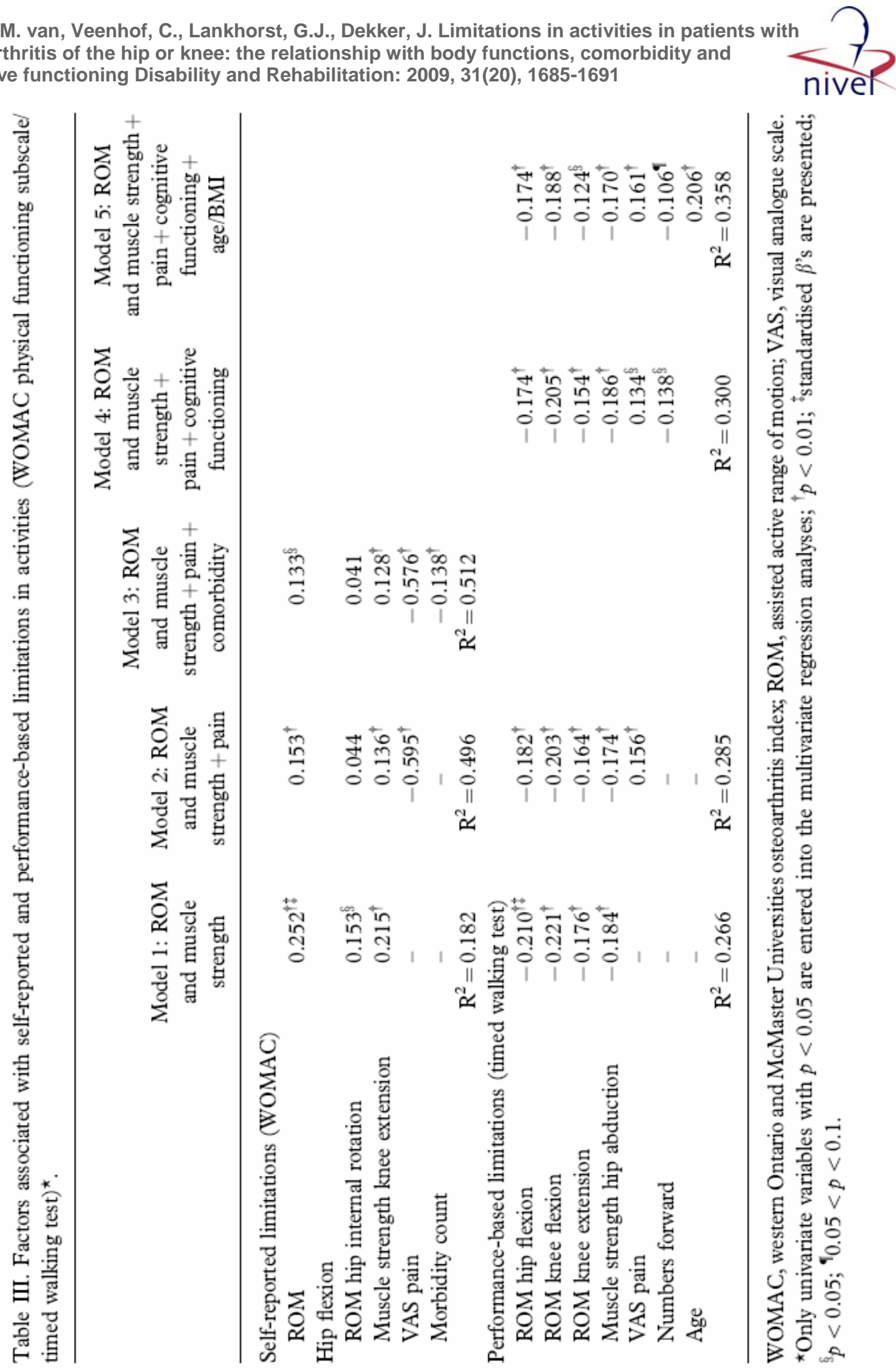
Box 1. Summary of associations found between body functions, comorbidity and cognitive functioning on the one side and more self-reported limitations in activities on the other side.

Factors associated with more

self-reported limitations

in activities

No association

Lower ROM hip flexion

Radiological impairments

Lower ROM hip internal rotation

ROM hip external rotation, knee

Lower muscle strength knee extension extension, knee flexion

More pain

Muscle strength in hip abduction

Higher morbidity count

Cognitive functioning

Age

BMI

ROM, assisted active range of motion; BMI, body mass index.

Box 2. Summary of associations found between body functions, comorbidity and cognitive functioning on the one side and more performance-based limitations in activities on the other side.

Factors associated with more performance-based limitations in activities No association

Lower ROM hip flexion

Lower ROM knee flexion

Lower ROM knee extension

Lower muscle strength hip abduction

More pain

Worse cognitive functioning

Older age
Radiological impairments ROM hip external rotation ROM hip internal rotation Muscle strength in knee extension

Morbidity count

BMI

ROM, assisted active range of motion; BMI, body mass index. 